\title{
Glossário - Termos do Modelo de House de avaliação de traduções para tradutores e estudiosos da tradução
}

\section{Glossary - Terms of House's Model of translation evaluation for translators and scholars of translation}

\author{
Márcia Ney Pessoa* \\ Cristiane Roscoe-Bessa*
}

Resumo: Este trabalho trata de um glossário em português dos termos do Modelo de House para avaliação de traduções. Para House, é necessário comparar o texto traduzido com o texto de partida para determinar se a tradução está adequada ou não aos objetivos do texto traduzido. House distingue a tradução explícita da tradução velada e apresenta estratégias que devem ser levadas em conta por quem avalia uma tradução. Os termos do glossário buscam elucidar as categorias de tradução e conceitos que dizem respeito a critérios para uma análise crítica da tradução de um gênero particular de texto e para a avaliação de traduções e conceitos que possibilitam caracterizar as consequências advindas da análise, em

\footnotetext{
" Mestra pela Universidade de Brasília (UnB).

"* Doutora pela Universidade de São Paulo (USP).
} 
termos de adequação ou não aos objetivos da tradução. Esta pesquisa pretende, também, demonstrar um modelo de glossário.

Palavras-chave: avaliação de traduções; Modelo de House; tradução explícita; tradução velada; modelo de glossário.

Abstract: This study is a Portuguese glossary of the terms of House's model for translation quality assessment. House supplies a comparative analysis of the target text and its source text. House argues that the model is based on the notion of equivalence, which is differentiated in her model according to an empirically derived distinction into overt and covert translation. The terms of the glossary define the categories of translation and other concepts concerning the criteria for assessing translation quality. This research also aims to demonstrate a glossary model.

Keywords: Translation Quality Assessment; House's Model; Overt translation; Covert translation; Glossary model. 
PessoA, M. N.; Roscoe-BesSA, C. - Glossário - Termos do Modelo de House de avaliação de traduções para tradutores e estudiosos da tradução

\section{Prefácio}

Muitos enunciados, se examinados com apuro, trazem palavras ou trechos de difícil compreensão, que necessitam de observação mais minuciosa. Muitas vezes uma consulta ao dicionário resolve o impasse. No entanto, há situações em que a dúvida persiste e requer elucidação. É isso o que um glossário objetiva. Conforme salienta Soares (2014), a construção de glossários é uma prática antiga. Remonta aos tempos dos manuscritos, em que anotações escritas entre uma linha e outra, ou em colunas nas margens das páginas, explicavam termos ou trechos que poderiam apresentar dificuldade de entendimento. Essas anotações recebiam o nome de glosas e colaboravam com os poucos leitores do passado, nos tempos que precederam a invenção da imprensa. Hoje, para colaborar com os muitos leitores, as glosas foram rebatizadas de verbetes e têm objetivo semelhante ao que tinham no passado: "a explicação de termos e conceitos que, porque complexos, ou porque recentemente introduzidos em uma área de conhecimento, ou ainda porque reconceituados pelo avanço dos conhecimentos, demandam ou merecem esclarecimento" (SOARES 2014). De acordo com Ferreira (2014: 64), um glossário "reúne, segundo Maria A. Barbosa, as palavras-ocorrência de um texto específico."

Neste sentido, analisar traduções com base no paradigma teórico dos Estudos Descritivos da Tradução (EDT), levando em consideração principalmente o Modelo de House (1997), suscita algumas questões, faz emergir pontos importantes para reflexão, termos que precisam ser explicados, que ainda necessitam de glosas para melhor compreensão. Este é o objetivo deste glossário. Ele pretende reunir, de forma breve e objetiva, os significados dos termos, expressões e palavras usadas pela teórica Juliane House na delineação de seu Modelo, nos capítulos 2 e 4 do livro Translation 
PessoA, M. N.; RosCOe-BessA, C. - Glossário - Termos do Modelo de House de avaliação de traduções para tradutores e estudiosos da tradução

quality assessment: a model revisited ${ }^{1}$. Tem como finalidade propor a conceituação ou reconceituaçao de tradução e de duas de suas categorias e respectivas características, além de alguns conceitos subjacentes à operacionalização da avaliação de traduções presentes nesses capítulos.

Assim, este glossário se destina a tradutores e estudiosos de tradução em diversos níveis e tem função descritiva, didática e também prescritiva. Ele pretende divulgar esse modelo de análise que tenta estabelecer regras e princípios gerais para a avaliação de traduções e enumerar e discutir exemplos concretos de problemas tradutórios. De acordo com House (1997: 124, 125, tradução nossa), "A tarefa de um modelo de avaliação de traduções é [...] analisar o mais objetivamente possível o que surgiu a partir do conjunto de circunstâncias no interior do qual a tarefa de tradução ou propósito foi concebido."2 Desse modo, sua realização se justifica por contribuir com a compreensão do Modelo de House (1997). Como esse Modelo discute problemas do conhecimento, ensejando algumas questões epistemológicas, ao apresentar a proposta teórica de criação de uma tipologia de tradução que envolve muitas outras questões relevantes para os estudos da área, a realização deste glossário pode vir a auxiliar a resolução de alguns problemas de tradução.

Ademais, este glossário, que compõe o texto de uma dissertação acerca da aplicação do Modelo, contém vinte e cinco termos. Devido à complexidade desse Modelo, que, além da análise de diversos aspectos linguísticos e estruturais dos textos, apresenta conceitos da área da análise do discurso, a inclusão do glossário no corpo do texto complementa as reflexões acerca da avaliação de traduções e possibilita uma análise mais profunda.

\footnotetext{
${ }^{1}$ Capítulo 2: "The original model for evaluating translations". Capítulo 4: "Refinement of the Model".

2 "The task of a model for translation quality assessment is [...] to assess as objectively as possible what has emerged from the set of circumstances inside which the translation task or purpose was conceived."
} 
PessoA, M. N.; RosCOe-BessA, C. - Glossário - Termos do Modelo de House de avaliação de traduções para tradutores e estudiosos da tradução

É importante salientar, ainda, que esse glossário é realizado sob a perspectiva teórico-metodológica da Teoria Comunicativa da Terminologia (TCT). Cabré (1993: 103, tradução nossa) ressalta, sobre a terminologia, que "a transferência de conhecimento é a sua dimensão comunicativa: a terminologia é a base da comunicação entre os profissionais." 3

\section{Introdução}

A discussão em pauta remete à aplicação do Modelo de House por meio da análise e comparação dos textos original e traduzido com vistas a determinar em que níveis eles são equivalentes, que tipo de tradução foi realizada e se essa tradução está adequada aos objetivos do texto traduzido. Ela compila os principais conceitos subjacentes à operacionalização dessa análise, presentes nos capítulos em que a teórica o explicita. Seu valor social consiste em contribuir para solucionar problemas de informação e comunicação. 0 conjunto de seus termos possibilita uma melhor comunicação entre pesquisadores e tradutores e viabiliza soluções para problemas com que possam vir a se deparar, uma vez que "várias significações de um termo coexistem dentro de uma mesma disciplina científica" (Hermans apud LaRA, 2004: 95). Além disso, por ser composto de termos traduzidos do inglês, o glossário contribui para o enriquecimento da língua portuguesa no que se refere ao discurso teórico epistemológico da área da tradução. Para Berman (2002 apud GoRovitz 2011: 15), ao re-produzir "o sistema-da-obra em sua língua, a tradução provoca nesta uma mudança."

Assim, a questão norteadora deste glossário diz respeito a identificar quais estratégias são válidas na tradução para que a função do texto original

\footnotetext{
3 "la transferencia del conocimiento constituye su dimensión comunicativa: la terminología es la base de la comunicación entre profesionales."
} 
PessoA, M. N.; Roscoe-BesSA, C. - Glossário - Termos do Modelo de House de avaliação de traduções para tradutores e estudiosos da tradução

seja mantida no texto traduzido, ou seja, para que a tradução seja, como define House (1997: 31, tradução nossa), "a re-contextualização de um texto na língua de partida por um texto semântica e pragmaticamente equivalente na língua de chegada." 4

Os termos vêm em português, em ordem alfabética, mas há em todos os verbetes o equivalente em inglês. Apesar de conter nomes, a grande maioria dos termos trata de fraseologismos, "objetos da Fraseologia marcados por seu caráter expressivo, sendo determinados por combinações de duas ou mais unidades lexicais" (FonseCA 2013: 30). Neste caso, será considerada a primeira letra da primeira palavra para a entrada. A macroestrutura é simples. O leitor pode se valer dos envios e referências cruzadas, pois são utilizadas remissivas para ampliar a compreensão. As remissivas implícitas apontam para sinônimos, ou seja, outros conceitos para as mesmas categorias, ou para conceitos presentes na definição que também são definidos no glossário. As remissivas explícitas indicam termos que têm entre si relação de oposição ou de complementaridade, além da página do livro de House (1997) de que se extraiu definição explícita.

Por fim, vêm notas em que os conceitos têm exemplos de aplicação prática. Para extrair do glossário o máximo rendimento, há um apêndice em que os termos são apresentados em inglês, em ordem alfabética, seguidos de sua tradução em português.

Na microestrutura, Albano (2009: 20) cita Almeida para se reportar às "informações sistemáticas (obrigatórias em todos os verbetes) e não sistemáticas (informações não-recorrentes)" presentes em verbetes. Assim, na microestrutura deste glossário vêm: o lema, informações linguísticas sobre esse lema, o equivalente em inglês, a definição e alguns contextos reais de uso, com exemplos extraídos da vivência das próprias pesquisadoras. Por fim,

\footnotetext{
4 “... replacement of a text in the source language by a semantically and pragmatically equivalent text in target language."
} 
PessoA, M. N.; Roscoe-BesSA, C. - Glossário - Termos do Modelo de House de avaliação de traduções para tradutores e estudiosos da tradução

conforme seja o caso, vêm termos que possuem relação de oposição ou de complementaridade com o lema.

Com relação aos aspectos dos termos deste glossário:

- Quanto à forma, os termos são fraseologismos (por exemplo, "tradução velada") e termos simples (por exemplo, "equivalência"). Não há termos compostos nem derivados;

- Quanto à função, os termos analisados são nomes ou sintagmas nominais;

- Sob o ponto de vista do significado, os termos são categorias de tradução, conceitos que dizem respeito a critérios para uma análise crítica da tradução de um gênero particular de texto e para a avaliação de traduções e conceitos que possibilitam caracterizar as consequências advindas da análise, em termos de adequação ou não aos objetivos da tradução.

\section{Estrutura do glossário}

Verbetes sempre são constituídos pela entrada e pela microestrutura. Esta, por sua vez, é formada pelos paradigmas. Barbosa (1990: 230) sugere "uma microestrutura com três macro paradigmas: paradigma informacional, paradigma definicional e paradigma pragmático" e que esses podem se subdividir, dependendo, entre outras coisas, do objetivo da obra lexicográfica.

Nesse sentido, na leitura vertical, o termo entrada vem em português, em ordem alfabética. Ele é apresentado na forma lematizada, isto é, substantivo masculino singular, substantivo feminino singular e fraseologismo. Exceção feita ao termo "relações", que ocorre na literatura especializada sobre tradução, em português, no plural, e que vêm dessa forma no glossário. 
PessoA, M. N.; RosCOe-BessA, C. - Glossário - Termos do Modelo de House de avaliação de traduções para tradutores e estudiosos da tradução

$\mathrm{Na}$ leitura horizontal, em seguida ao termo entrada, na mesma linha, no paradigma informacional é apresentada a referência gramatical do termo. Em letra minúscula, os substantivos masculinos são assinalados por "s.m.", os femininos por "s.f." e os fraseologismos pela classificação do seu núcleo, o substantivo, igualmente, "s.m." e "s.f". Na segunda linha é apresentada a equivalência em inglês.

No paradigma definicional são descritas as funções de cada um dos conceitos relativamente à análise de traduções. As definições são do tipo terminológicas. Quando o termo diz respeito a um critério, dimensão, requisito ou objetivo a ser atingido, há numeração expressa (1., 2.) na definição. A definição contida no número 1 refere-se à definição terminológica do termo e a segunda denota o conceito do qual o termo é critério, dimensão ou requisito.

Está presente também o paradigma pragmático, em que contextos reais de uso são demonstrados. Também caracteriza esse paradigma a presença de remissivas. Estas vêm em forma de asterisco $\left({ }^{*}\right)$ e o conceito a que remetem, no caso, sinônimos, imediatamente após as referências linguísticas, em lugar da definição. Quando em uma definição estiver presente algum termo cuja definição também é apresentada no glossário, este termo vem destacado (em negrito). Os exemplos de aplicação das definições vêm em forma de nota, depois da definição, precedidos da palavra "nota". Esses usos advêm da prática das pesquisadoras. Entre parênteses, sublinhado, vêm abonações. Por fim, as remissivas explícitas, quando houver, vêm no final do verbete, depois de "cf.", e indicam termos que têm entre si relação de oposição ou de complementaridade. Ressalte-se ainda, que, sempre que expresso, o número entre colchetes no final de cada verbete refere-se à página do livro de House (1997) da qual a definição terminológica foi extraída.

Todas essas questões refletem-se diretamente na forma de apresentação do glossário, que vem a seguir. 
PessoA, M. N.; Roscoe-BesSA, C. - Glossário - Termos do Modelo de House de avaliação de traduções para tradutores e estudiosos da tradução

\section{Glossário}

avaliação de traduções s.f.

translation quality assessment

análise linguística e comparação sistemática entre o texto original e o traduzido e julgamento do seu valor social. Nota 1: A avaliação de traduções proposta no Modelo de House tem por base a teoria sistêmico-funcional de Halliday, a análise do discurso e as teorias pragmáticas de uso da língua. Nota 2: 0 modelo proposto por House para essa análise não tem por objetivo fornecer critérios absolutos de julgamento. Nota 3: 0 Modelo também não aponta para juízos de valor como "boas" ou "ruins" para traduções, mas para a observação de quais fatores comunicativos concorrem para a consecução da equivalência. Nota 4: A avaliação de traduções, no Modelo de House, tem uma abordagem linguística.

campo s.m.

field
1. assunto ou tema de um texto. 2. Uma das dimensões do registro. Nota: 0 texto é a história de uma família de ursos sob a forma de um livro ilustrado. (Relativamente a texto analisado por House, 2015, p. 71) Cf. Teor Cf. Modo [108] contexto situacional s.m. context of situation aspectos da realidade extralinguística relevantes para a compreensão de um texto. Nota: "Bye, Felicia!" $\rightarrow$ (Em diálogo com uma pessoa chamada Felícia) "Tchau, Felícia!" (Em alguns outros contextos, dependendo da entonação) “Já vai tarde!” [37] domínio s.f. *campo equivalência s.f. equivalence

1. medida em que textos têm $o$ mesmo sentido em línguas diferentes. 2. Critério principal de qualidade de uma tradução. Nota: "It's snowing." $\rightarrow$ "Está nevando." [30]

equivalência estético-formal s.f. 
PessoA, M. N.; Roscoe-BesSA, C. - Glossário - Termos do Modelo de House de avaliação de traduções para tradutores e estudiosos da tradução

formal-aesthetic equivalence

1. medida em que a forma estética e o efeito de um texto original é transposta para sua tradução. 2. Objetivo da tradução explícita. Nota: "Blood, toil, tears, and sweat." $\rightarrow$ Traduzir, por exemplo, como "Sangue, labuta, lágrimas, e suor.", em vez da tradução amplamente divulgada, "Sangue, suor e lágrimas." (Trecho de um discurso político proferido por Winston Churchill em 1942) Cf. Equivalência [25]

equivalência funcional s.f.

functional equivalence

1. medida em que um texto traduzido funciona, em um contexto situacional, da mesma maneira que o original. 2. Objetivo da tradução velada. Nota: "Easy does it." $\rightarrow$ "Vá com calma." (Tradução de um lema da irmandade Alcoólicos Anônimos) Cf. Equivalência [29]

equivalência funcional de segundo nível s.f.

second-level functional equivalence tipo de equivalência remota característico da tradução explícita em que texto original e traduzido nunca têm "o mesmo" sentido para seus respectivos leitores. Nota: a função da tradução explícita é possibilitar aos leitores o acesso à função do texto original em seu ambiente cultural e linguístico. Nesse sentido, House (1997) salienta que, ao traduzir um texto como um sermão de Karl Barth, proferido para prisioneiros de Basel, na Suíça, em 1964, por exemplo, 0 tradutor precisa viabilizar aos membros da cultura de chegada acesso ao texto original e seu impacto cultural sobre os membros da cultura de partida. Cf. Equivalência [67]

filtro cultural s.m.

cultural filter

meio de identificar e eliminar as diferenças culturais entre a comunidade da língua de origem e a da língua da tradução. Nota: "Dear Parents" (Em manual de uma cadeira infantil para veículos fabricada na Alemanha) $\rightarrow$ "Senhores Pais" (As normas 
PessoA, M. N.; RosCOe-BessA, C. - Glossário - Termos do Modelo de House de avaliação de traduções para tradutores e estudiosos da tradução

culturais para o emprego de Dear / Querido são diferentes no inglês e no português do Brasil). [71]

função textual s.f.

textual function

1. aplicação ou uso que um texto tem no contexto particular de uma situação, consistindo num exemplo de gênero. 2. Requisito principal de equivalência funcional. Nota: A função de um texto é aquela que seu próprio nome designa. Por exemplo, um rótulo traduzido deve funcionar como um rótulo comercial para o consumidor da comunidade de chegada. [36]

gênero s.m.

genre

tipo de evento comunicativo (como um sermão ou um trabalho acadêmico) que obedece a convenções socioculturais, se realiza textualmente por diferentes configurações lexicais e gramaticais e resulta de diferentes usos sociais e contextos de produção. $C f$. Registro [107]

modo s.m.

mode
1. uso do canal ou meio de comunicação na fala e na escrita 2. Uma das dimensões do registro. Nota: Texto escrito para ser lido (relativamente a um manual de assento infantil para automóveis). Cf. Campo Cf. Teor [109] registro s.m. register variedade da língua de acordo com seu uso em determinados contextos ou de acordo com o gênero textual. Nota: Um livro escrito para crianças precisa do emprego de uma linguagem que crianças possam compreender. [105]

relação de duplo vínculo s.f

double-bind relationship

fato pelo qual toda tradução é vinculada tanto ao seu texto original quanto às condições de sua recepção no contexto de chegada.

Nota: É necessário investigar o que o texto original fez em seu cenário original para determinar como ele deve ser traduzido. [29]

relações s.f.pl *teor

segundo original s.m.

second original 
PessoA, M. N.; Roscoe-BesSA, C. - Glossário - Termos do Modelo de House de avaliação de traduções para tradutores e estudiosos da tradução

posição que um texto traduzido de forma velada ocupa na cultura de chegada.

Nota: Um rótulo traduzido funciona como um texto local, como se tivesse sido produzido a partir de e para a cultura de chegada. [69]

teor s.m.

tenor

1. relacionamento entre os participantes no uso da língua. 2. Uma das dimensões do registro. Nota: Um texto pode ter características que indicam solidariedade $e$ igualdade entre remetente e destinatários ou apresentar relação de autoridade entre eles. Cf. Campo Cf. Modo [108; 109]

texto s.m.

text

qualquer extensão da língua em que os componentes individuais se relacionam entre si e formam um conjunto coeso. Nota: Frase, manual de uma cadeira infantil para uso em veículos, poema, romance. [31]

tradução s.f.

\section{translation}

substituição de um texto na língua de origem por um texto semântica e pragmaticamente equivalente na língua de chegada. Nota: Há três aspectos particularmente importantes para a tradução: o semântico, o pragmático e 0 textual. [31]

tradução encoberta s.f. * tradução velada

tradução evidente s.f. ${ }^{*}$ tradução explícita

\section{tradução explícita s.f.}

overt translation

tipo de tradução em que as características culturais do texto original são intencionalmente mantidas. Nota: A tradução é como se fosse uma "citação" do texto original. Ela é explicitamente algo "estrangeiro", "estranho" para o leitor, pois o texto original não sofre grandes alterações. Por exemplo, de acordo com House (1997), os leitores do coreano podem utilizar um texto de chegada de um discurso de Churcill para terem acesso ao texto de 
PessoA, M. N.; Roscoe-BesSA, C. - Glossário - Termos do Modelo de House de avaliação de traduções para tradutores e estudiosos da tradução

partida, mas sabem, pelo texto, que leem uma tradução, não um texto escrito em coreano. $C f$. Tradução velada [66]

tradução manifesta s.f. * tradução explícita

tradução ostensiva s.f. * tradução explícita

tradução velada s.f.

covert translation

tipo de tradução realizada a fim de que $\mathrm{o}$ texto traduzido se mostre como se tivesse sido produzido na cultura de chegada com a utilização de um filtro de cultural. Nota: Exemplos de tradução velada tal como preconizada por House (1997), incluem textos como manuais e rótulos, e são apresentados aos leitores como se tivessem sido produzidos na própria língua de chegada, sem qualquer referência a um texto de partida que tenha servido de referência para a tradução. Cf. Tradução explícita [69]

\section{Exemplos de fichas terminológicas}

A pesquisa se deu na sequência a seguir: 1) leitura rápida do capítulo; 2) tradução do capítulo; 3) realização do fichamento bibliográfico (em português); 3) identificação dos termos por meio da análise do que a teórica propõe para seu Modelo; 4) elaboração das fichas de citação; 5) elaboração das fichas terminológicas; 6) elaboração do glossário.

Os dados coletados foram registrados nas fichas de recolha que, segundo Barros (2004: 211), registram "a unidade terminológica, a fonte (referência bibliográfica completa) [...] o contexto e a ilustração." Cumpre ressaltar que, como o trabalho diz respeito a um glossário, na ficha de recolha, em vez da referência bibliográfica completa, colocou-se a página em que a unidade terminológica aparece pela primeira vez e, conforme seja a situação, a página em que ocorre uma definição explícita. No entanto, por 
PessoA, M. N.; Roscoe-BesSA, C. - Glossário - Termos do Modelo de House de avaliação de traduções para tradutores e estudiosos da tradução

vezes, as definições não vêm expressas, mas são descritas analiticamente. A partir desse ponto, imediatamente em seguida, foram elaboradas as fichas terminológicas, com a inclusão dos demais dados. As fichas de recolha foram numeradas na ordem de identificação de cada termo e as terminológicas repetem esse número, uma vez que são o desenvolvimento daquelas. Na ficha terminológica, o metadado "unidade terminológica" passa a ser referido como "entrada".

Barros (2004: 211) faz distinção entre dados terminológicos e dados terminográficos. Os primeiros referem-se "à unidade terminológica, sua descrição linguística e às relações que o termo mantém com as outras unidades terminológicas estudadas". Já os dados terminográficos "oferecem precisões sobre a unidade terminológica estudada, indicações sobre o tratamento terminográfico."

4.1. Exemplo de ficha de recolha fraseologismo feminino

\begin{tabular}{|c|c|}
\hline FICHA & 9 \\
\hline $\begin{array}{l}\text { UNIDADE } \\
\text { TERMINOLÓGICA }\end{array}$ & FUNÇÃO TEXTUAL \\
\hline DEFINIÇÃO & $\begin{array}{l}\text { 1. Aplicação ou uso que um texto tem no contexto particular } \\
\text { de uma situação, consistindo num exemplo de gênero. } 2 \text {. } \\
\text { Requisito principal de equivalência. (\#equivalência } \\
\text { funcional) }\end{array}$ \\
\hline PÁGINA & $32 ; 36$ \\
\hline APLICAÇÃO & $\begin{array}{l}\text { A função de um texto é aquela que seu próprio nome } \\
\text { designa. Por exemplo, um rótulo traduzido deve funcionar } \\
\text { como um rótulo comercial para o consumidor da comunidade } \\
\text { de chegada. }\end{array}$ \\
\hline
\end{tabular}

\subsection{Exemplo 1 de ficha terminológica - fraseologismo masculino}


PessoA, M. N.; Roscoe-BesSA, C. - Glossário - Termos do Modelo de House de avaliação de traduções para tradutores e estudiosos da tradução

\begin{tabular}{|c|c|}
\hline FICHA & 10 \\
\hline ENTRADA & CONTEXTO SITUACIONAL \\
\hline $\begin{array}{l}\text { CLASSIFICAÇÃO } \\
\text { GRAMATICAL }\end{array}$ & Fraseologismo masculino \\
\hline DEFINIÇÃO & $\begin{array}{l}\text { Aspectos da realidade extralinguística relevantes para a } \\
\text { compreensão de um texto. }\end{array}$ \\
\hline PÁGINA & 37 \\
\hline APLICAÇÃO & 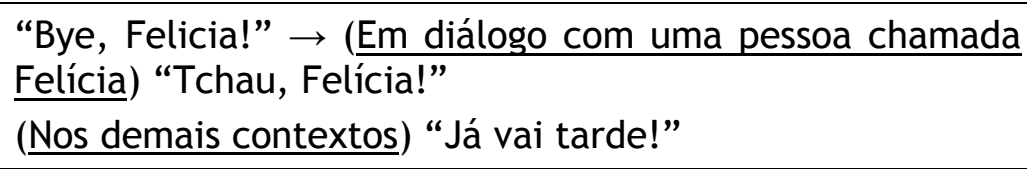 \\
\hline EQUIVALENTE & Context of situation \\
\hline \multicolumn{2}{|l|}{ SINÔNIMO } \\
\hline REMISSIVA & {$[37]$} \\
\hline DATA DA COLETA & 23 jun. 2015 \\
\hline AUTORA & Márcia Ney Pessoa \\
\hline
\end{tabular}

\subsection{Exemplo 2 de ficha terminológica - fraseologismo feminino}

\begin{tabular}{|c|c|}
\hline FICHA & 4 \\
\hline ENTRADA & TRADUÇÃO VELADA \\
\hline $\begin{array}{l}\text { CLASSIFICAÇÃO } \\
\text { GRAMATICAL }\end{array}$ & Fraseologismo feminino \\
\hline DEFINIÇÃO & $\begin{array}{l}\text { Tipo de tradução realizada a fim de que o texto traduzido } \\
\text { se mostre como se tivesse sido produzido na cultura de } \\
\text { chegada através da utilização de um filtro cultural. }\end{array}$ \\
\hline PÁGINA & $29 ; 69$ \\
\hline APLICAÇÃO & $\begin{array}{l}\text { “I am a teacher." } \rightarrow \text { "Sou professora." ( } 0 \text { filtro cultural } \\
\text { mostra que, no uso corrente, em português, há elipse do } \\
\text { sujeito quando as desinências verbais permitem sua } \\
\text { identificação. Da mesma forma, a tendência é a supressão } \\
\text { dos artigos, a não ser em casos específicos.) }\end{array}$ \\
\hline EQUIVALENTE & Covert translation \\
\hline
\end{tabular}


PessoA, M. N.; Roscoe-BesSA, C. - Glossário - Termos do Modelo de House de avaliação de traduções para tradutores e estudiosos da tradução

\begin{tabular}{|l|l|}
\hline SINÔNIMO & \\
\hline REMISSIVA & Tradução explícita; [66] \\
\hline DATA DA COLETA & 20 jun. 2015 \\
\hline AUTORA & Márcia Ney Pessoa \\
\hline
\end{tabular}

4.4. Exemplo 3 de ficha terminológica - nome masculino

\begin{tabular}{|l|l|}
\hline FICHA & $\mathbf{7}$ \\
\hline ENTRADA & TEXTO \\
\hline $\begin{array}{l}\text { CLASSIFICAÇÃO } \\
\text { GRAMATICAL }\end{array}$ & Substantivo masculino \\
\hline DEFINIÇÃO & $\begin{array}{l}\text { Qualquer extensão da língua em que os componentes } \\
\text { individuais se relacionam entre si e formam um conjunto } \\
\text { coeso. }\end{array}$ \\
\hline PÁGINA & 29; 31 \\
\hline APLICAÇÃO & $\begin{array}{l}\text { Frase, manual de uma cadeira infantil para uso em } \\
\text { veículos, poema, romance. }\end{array}$ \\
\hline EQUIVALENTE & Text \\
\hline SINONIMO & {$[31]$} \\
\hline REMISSIVA & 30 jun. 2015 \\
\hline DATA DA COLETA & Márcia Ney Pessoa \\
\hline AUTORA &
\end{tabular}

\subsection{Exemplo 4 de ficha terminológica - substantivo feminino}

\begin{tabular}{|l|l|}
\hline FICHA & 1 \\
\hline ENTRADA & EQUIVALÊNCIA \\
\hline $\begin{array}{l}\text { CLASSIFICAÇÃO } \\
\text { GRAMATICAL }\end{array}$ & Substantivo feminino \\
\hline
\end{tabular}


PessoA, M. N.; Roscoe-BesSA, C. - Glossário - Termos do Modelo de House de avaliação de traduções para tradutores e estudiosos da tradução

\begin{tabular}{|l|l|}
\hline DEFINIÇÃO & $\begin{array}{l}\text { 1. medida em que textos têm o mesmo sentido em línguas } \\
\text { diferentes 2. Critério principal de qualidade de uma } \\
\text { tradução. }\end{array}$ \\
\hline PÁGINA & $29 ; 31$ \\
\hline APLICAÇÃO & “It's snowing." $\rightarrow$ “Está nevando.” \\
\hline EQUIVALENTE & Equivalence \\
\hline SINONIMO & \\
\hline REMISSIVA & {$[30]$} \\
\hline DATA DA COLETA & 20 jun. 2015 \\
\hline AUTORA & Márcia Ney Pessoa \\
\hline
\end{tabular}

\section{Considerações finais}

Este artigo apresenta um glossário de termos, expressões e palavras empregadas por House (1997) na delineação de seu Modelo de avaliação de traduções. Ele tencionou contribuir com a compreensão desse Modelo, ampliando o discurso epistemológico da tradução em língua portuguesa. A pesquisa pretendeu, também, demonstrar um modelo de glossário e de fichas terminológicas, de acordo com a Teoria Comunicativa da Terminologia.

Além de o glossário apontar para a padronização da tradução dos termos do Modelo de House (1997), uma vez que fez uma escolha entre as traduções de um mesmo termo, já existentes em português, ele compila em um só artigo os principais conceitos subjacentes à operacionalização da avaliação de traduções proposta por House (1997). Para mais, o estudo traz algumas notas que exemplificam a aplicação das definições e demonstram empregos do termo conforme a categorização proposta no glossário.

Ademais, sugerem-se novas pesquisas, tanto para a ampliação do discurso epistêmico da tradução em língua portuguesa e para a solidificação dos conceitos apresentados, como para sua discussão. 
PessoA, M. N.; Roscoe-BesSA, C. - Glossário - Termos do Modelo de House de avaliação de traduções para tradutores e estudiosos da tradução

\section{Referências bibliográficas}

Albano, N. M. Macro and Micro Structure of the Terminological Glossary of Aromatherapy (Glotear). In: UNOPAR Cient., Ciênc. Human. Educ., v. 10, n. 2. Londrina, out. 2009. p. 17-22.

BARBOSA, M. A. Lexicologia, Lexicografia, Terminologia, Terminografia: identidade científica, o objeto, métodos e campos de atuação. II Simpósio LatinoAmericano de Terminologia. Brasília, 1990. p. 152-153.

BARRoS, L. A. Curso básico de terminologia. São Paulo: EdUSP, 2004.

CABRÉ, M. T. La terminologia: teoria, metodologia, aplicaciones. Barcelona: Antártida/Empúries, 1993.

FerReira, A. M. de A. Existem dicionários de tudo e o tradutor sabe disso!!?? In: Ferreira, A. M. de A.; SousA, G. H. P. de; Gorovitz, S. (Orgs.) A tradução na sala de aula: ensaios de teoria e prática de tradução. Brasília: Editora da Universidade de Brasília, 2014. p. 55-72.

FonseCA, H. da C. Fraseologismos zoônimos: elaboração de base de dados português-francês. Dissertação (Mestrado) - Instituto de Biociências, Letras e Ciências Exatas da Universidade Estadual Paulista. São José do Rio Preto, 2013.

GoRovitz, S. A tradução enquanto situação de passagem. In: BeLl-SANTOS, C. A.; Roscoe-Bessa, C.; Hatje-Faggion, V.; Sousa, G. H. P. (Orgs.) Tradução e cultura. Rio de Janeiro: 7Letras, 2011. p. 13-22.

HOUSE, J. Translation quality assessment: a model revisited. Tübingen: Narr, 1997. 
PessoA, M. N.; Roscoe-BesSA, C. - Glossário - Termos do Modelo de House de avaliação de traduções para tradutores e estudiosos da tradução

LaRA, M. L. G. de. "Diferenças conceituais sobre termos e definições e implicações na organização da linguagem documentária". In: Ciência da Informação, v. 33, n. 2, Brasília. p. 91-96.

SOARES. M. B. "Prefácio: A que serve um glossário? Por que e para que um glossário? Glossário Ceale”. Belo Horizonte: 2014. Disponível em <http://ceale.fae.ufmg.br/app/webroot/glossarioceale/prefacio>. Último acesso: 30 maio 2015.

\section{Apêndice 1}

\section{Apresentação dos termos em inglês}

CONTEXT OF SITUATION: contexto situacional

COVERT TRANSLATION: tradução velada

CULTURAL FILTER: filtro cultural

DOUBLE-BIND RELATIONSHIP: relação de duplo vínculo

EQUIVALENCE: equivalência

FIELD: campo

FORMAL-AESTHETIC EQUIVALENCE: equivalência estético-formal

FUNCTIONAL EQUIVALENCE: equivalência funcional

GENRE: gênero

MODE: modo

OVERT TRANSLATION: tradução explícita

REGISTER: registro 
PessoA, M. N.; Roscoe-BesSA, C. - Glossário - Termos do Modelo de House de avaliação de traduções para tradutores e estudiosos da tradução

SECOND-LEVEL FUNCTIONAL EQUIVALENCE: equivalência funcional de segundo nível

SECOND ORIGINAL: segundo original

TENOR: teor

TEXT: texto

TEXTUAL FUNCTION: função textual

TRANSLATION: tradução

TRANSLATION QUALITY ASSESSMENT: avaliação de traduções

Data de submissão: 23/10/2016

Data de aprovação: 19/05/2017 